\title{
Homeostatic Modulation of Stimulation-Dependent Plasticity in Human Motor Cortex
}

\author{
N. V. ILIĆ ${ }^{1}$, S. MILANOVIĆ ${ }^{2}$, J. KRSTIĆ ${ }^{3}$, Đ. D. BAJEC ${ }^{4}$, M. GRAJIĆ ${ }^{1}$, T. V. ILIĆ ${ }^{5}$ \\ ${ }^{1}$ Clinic of Rehabilitation Medicine, Clinical Center of Serbia, ${ }^{2}$ Institute of Medical Research, \\ University of Belgrade, ${ }^{3}$ Clinical Center "Dragiša Mišović", ${ }^{4}$ Clinical Center of Serbia, Belgrade, \\ ${ }^{5}$ Department of Clinical Neurophysiology, Military Medical Academy, Belgrade, Serbia
}

Received December 3, 2010

Accepted March 30, 2011

On-line July 19, 2011

\section{Summary}

Since recently, it is possible, using noninvasive cortical stimulation, such as the protocol of paired associative stimulation (PAS), to induce the plastic changes in the motor cortex, in humans that mimic Hebb's model of learning. Application of TMS conjugated with peripheral electrical stimulation at strictly coherent temporal manner lead to convergence of inputs in the sensory-motor cortex, with the consequent synaptic potentiation or weakening, if applied repetitively. However, when optimal interstimulus interval (ISI) for induction of LTP-like effects is applied as a single pair, Motor evoked potential (MEP) amplitude inhibition is observed, the paradigm known as short-latency afferent inhibition (SLAI). Aiming to resolve this paradox, PAS protocols were applied, with 200 repetitions of TMS pulses paired with median nerve electrical stimulation, at ISI equal to individual latencies of evoked response of somatosensory cortex $\left(\mathrm{N}_{20}\right)$ (PAS $\mathrm{LTP}_{\mathrm{T}}$, and at ISI of $\mathrm{N}_{20}$ shortened for $5 \mathrm{msec}\left(\mathrm{PAS}_{\mathrm{LTD}}\right)$ protocols that mimic LTP-like changes in the human motor cortex. MEP amplitudes before, during and after interventions were measured as an indicator based on output signals originating from the motor system. Post-intervention MEP amplitudes following the TMS protocols of PAS $\mathrm{LTP}_{\mathrm{TP}}$ and PAS $\mathrm{STD}_{\mathrm{TT}}$ were facilitated and depressed, respectively, contrary to MEP amplitudes during intervention. During PAS LTP MEP amplitudes

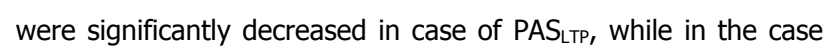
of PAS $\mathrm{PLD}_{\mathrm{LT}}$ an upward trend was observed. In conclusions, a possible explanation for the seemingly paradoxical effect of PAS can be found in the mechanism of homeostatic modulation of plasticity. Those findings indicate the existence of complex relationships in the development of plasticity induced by stimulation, depending on the level of the previous motor cortex excitability.

\section{Key words}

Transcranial magnetic stimulation • Paired associative stimulation - Motor cortex • Homeostatic plasticity

\section{Corresponding author}

T.V. Ilic, Dept of Clin Neurophysiology, $4^{\text {th }}$ floor, r. 52, Military Medical Academy, Crnotravska 17, 11000 Belgrade, Serbia. Fax: ++ 381113608.745 . E-mail: tihoilic@gmail.com

\section{Introduction}

Neural plasticity represents the ability of neural circuits to undergo changes in function or organization due to previous activity. A modification of presynaptic inputs, can cause extended activity-dependent changes of efficacy of excitatory or inhibitory synaptic connections between neurons, known as synaptic plasticity (Citri and Malenka 2008). As it was shown in animal models, longterm potentiation (LTP) of synaptic transmission is a long-lasting, activity-dependent form of synaptic plasticity that can be induced experimentally by applying a sequence of short, high-frequency stimulations to presynaptic neurons (Bliss and Collinridge 1993). Longterm depression (LTD), contrary to LTP, is characterized by a decrease in synaptic efficacy (Rothwell 1997). According to the learning rule introduced by Hebb, the synaptic connection between two neurons is strengthened if the firing of the presynaptic neuron is repeatedly and persistently paired with firing of the postsynaptic neuron, the process known as associative plasticity (Hebb 1949). However, prolonged periods of synaptic activity tend to drive networks into either a maximized or a minimized 
state of neuronal firing, that needs a sort of "gain control" in order to maintain physiological functions within a set narrow range (homeostatic plasticity). Since the introduction of transcranial magnetic stimulation (TMS), in the mid 1980s it became possible to stimulate cortical regions with repetitive pulses of TMS that provide us an opportunity to replicate animal findings through noninvasive, neurophysiological studies on humans. The introduction of a paradigm of paired associative stimulation (PAS) that couples TMS over motor cortex preceded by electrical stimuli delivered on a peripheral nerve in strict temporal coherence led us to the possibility of exploring LTP- or LTD-like phenomena in the human motor cortex (Stefan et al. 2000). When afferent sensory input from peripheral nerve electrical stimulation reaches the motor cortex at the time of magnetic pulse delivery, PAS induces LTP-like facilitation of MEP amplitudes in the target muscle. Contrary, if the magnetic pulse is delivered over scalp prior to arrival of sensory input to the cortex, PAS changes the direction of synaptic modification, inducing LTD-like plasticity (Stefan et al. 2002).

However, contrary to repeated stimulation with two coherent stimuli in PAS paradigm (200 pairs), when electrical shock to the median nerve at wrist was given at ISIs between 19 and $21 \mathrm{~ms}$ before TMS, in single pair, MEP amplitudes were suppressed, a phenomenon called short latency afferent inhibition (SLAI) (Tokimura et al. 2000). At the same ISI, PAS paradigm with repetitive associative stimulation produces opposite effect, postintervention MEP amplitude augmentation. To our knowledge, previous studies were focused mainly on the difference between the MEP amplitude before and after the intervention, but tracking changes in MEP amplitude during the interventional period, has not been analyzed (Stefan et al. 2000, Ziemann et al. 2004). In accordance with known effects of a single pair of stimuli in SLAI paradigm, we have performed this study aiming to evaluate the possibility that PAS interventional protocols move the threshold of neuronal activation in the opposite direction of the changes which are observed during postintervention follow-up period.

\section{Methods}

Experiments were performed on 14 right-handed healthy volunteers ( 5 female) between 24 and 47 years of age (mean $36.9 \pm 8.1$ years). None had a history of neurological disease or were on CNS-active drugs at the time of the experiments. All subjects gave their written informed consent for participation in the study. The study was approved by the local Ethical Committee of the Military Medical Academy, Belgrade. The experiments conformed to the Declaration of Helsinki.

\section{EMG recording}

During the experiment, subjects were comfortably seated in an armchair with their hands supported by armrests. Surface electromyographic (EMG) recordings in a belly-tendon montage were made from the right abductor pollicis brevis (APB) muscle using $\mathrm{Ag}-\mathrm{AgCl}$ electrodes (diameter $9 \mathrm{~mm}$ ). The raw EMG signal was amplified and filtered with a bandpass filter range of $20 \mathrm{~Hz}$ to $1 \mathrm{kHz}$ (MS91 Medelec, Medelec, UK). Signals were digitized at $5 \mathrm{kHz}$ (CED 1401 plus, Cambridge Electronic Design, Cambridge, UK) and stored on a computer for subsequent off-line analysis.

\section{Somatosensory evoked potentials}

Median-nerve somatosensory evoked potentials were recorded according to international guidelines (Cruccu et al. 2008) using surface electrodes. The active electrode was placed over the skull region overlying the primary somatosensory cortex (C3' using the international 10-20 system) while the reference electrode was placed over frontal midline position $(\mathrm{Fz})$. For each of a minimum of three reproductions, 1024 electrical stimuli (pulse width $300 \mu \mathrm{s}, 3 \mathrm{~Hz}, 10-20 \mathrm{~mA}$ ) were applied to the contralateral median nerve.

\section{Transcranial magnetic stimulation (TMS)}

TMS was performed using a Magstim 200 stimulator with a monophasic current waveform (Magstim Co, Dyfed, UK) connected to a figure-of-eightshape coil. The coil was held over the presumed scalp projection of primary motor cortex (M-1) with a handle pointing backwards and laterally approximately 45 degrees to the inter-hemispheric line to induce an anteriorly directed current in the brain. This is the optimal orientation for activating the corticospinal system transsynaptically via horizontal cortical connections (Sakai et al. 1997). The coil was optimally positioned to evoke MEPs in the right APB muscle.

The resting motor threshold (RMT) was defined as a minimal stimulator output intensity that evoked a MEP of $\geq 50 \mu \mathrm{V}$ in five out of ten consecutive trials (Chen et al. 2008). The intensity of magnetic stimulation was then adjusted to induce approximate peak-to-peak 



A-P $4^{\circ}$


$10 \mathrm{~min}$

20 min

$30 \mathrm{~min}$

Fig. 1. Time line of experiments (for details, see Methods).

amplitude of $1 \mathrm{mV}$ in the resting $\mathrm{APB}$, when given without the preceding median nerve stimulus.

\section{Paired associative stimulation (PAS)}

PAS consisted of 200 electrical stimuli of the right median nerve at the wrist, each paired with consecutive TMS over of the hand area of the left M-1, at fixed ISI. The rate of paired stimulation was $0.25 \mathrm{~Hz}$ thus taking about 15 minutes to complete. Electrical stimulation was applied through a bipolar electrode (cathode proximal) using a constant current square wave pulse (duration, $1 \mathrm{~ms}$ ) at an intensity of 3 times the perceptual threshold (range 0.75-3.45 mA). Inter-stimulus interval between the median nerve stimulus and TMS were individually adjusted based on the $\mathrm{N}_{20}$ cortical component of the median nerve somatosensory evoked potential (Ziemann et al. 2004). For this reason, the ISIs for each subject were adapted to the individual $\mathrm{N}_{20}$ latency, in order to enhance LTP-like changes in MEP amplitude in the target muscle $\left(\mathrm{PAS}_{\mathrm{LTP}}\right)$. In order to induce LTD-like effects ISI of $\mathrm{N}_{20}-5$ msec was used (PAS $\mathrm{PTD}_{\mathrm{LT}}$. The values of $\mathrm{N}_{20}$ cortical latencies were in range $18.7-21.0 \mathrm{~ms}$.

\section{Data analysis}

Relaxation of the APB was monitored audiovisually with high gain EMG $(50 \mu \mathrm{V} /$ div $)$. Trials contaminated with voluntary EMG activity were discarded from analysis.

Resting motor threshold (RMT) was measured before and (baseline, time point B) immediately after (post-interventional time $1, \mathrm{P}_{1}$ ) to check if any of two PAS protocols induced changes in MEP amplitude that may confound further analysis. At each time point, $20 \mathrm{MEP}$ were obtained at a mean inter-trial interval of $10 \mathrm{~s}$ and a random inter-trial interval variation of $25 \%$. For each subject and time point, the single-trial peak-topeak MEP amplitudes were averaged and normalized to the MEP amplitude measured at baseline.

MEP amplitudes were measured before PAS (baseline, time point $\mathrm{B}$ ), immediately after $\left(\mathrm{P}_{1}\right)$, and $5 \min \left(\mathrm{P}_{2}\right), 10 \min \left(\mathrm{P}_{3}\right), 15 \min \left(\mathrm{P}_{4}\right), 20 \min \left(\mathrm{P}_{5}\right)$ and 30 min later $\left(\mathrm{P}_{6}\right)$ in order to assess changes in left $\mathrm{M} 1$ (Fig. 1). Furthermore MEP amplitudes that were registered during interventional protocols were split in bins of 10 MEPs (total of 20 bins) in order to study the time changes. The MEP amplitude reflects synaptic excitability in $\mathrm{M}-1$, which is regulated through various inhibitory and excitatory neurotransmitter systems (Boroojerdi et al. 2001).

MEP amplitude means were calculated for each time bin, including baseline values. The MEPs during interventions as well as post-intervention MEPs were normalized and are given as ratios of the baseline determined immediately before intervention.

Regarding the criteria for successful intervention, we have accepted the criteria derived from animal studies that marked LTP or LTD respectively as a facilitation or depression of the amplitude of postsynaptic response of at least $10 \%$, which is also represented by more than $2 \mathrm{SD}$ as compared to the baseline amplitude (Hess and Donoghue 1999).

Changes in MEPs induced by PAS protocols were averaged over time points $\mathrm{P}_{1}$ and $\mathrm{P}_{6}$ and compared to MEPs before the intervention (B) using a two-tailed paired t-test. To test for effects of group, a repeated 


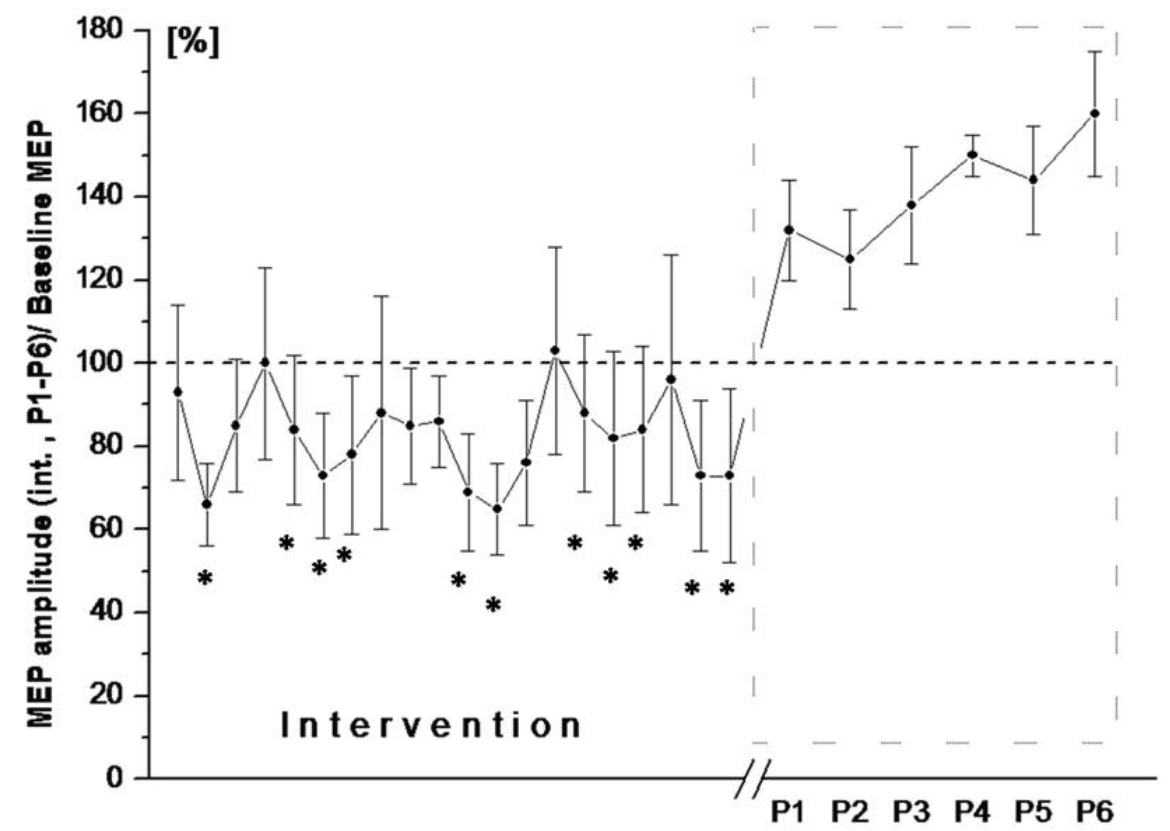

Fig. 2. Effects of $P A S_{L T P}$ on MEPS amplitude during intervention and post intervention in the resting abductor pollicis brevis (APB) muscle. The PAS intervention took about 15 minutes to complete. All interventional data are binned in 10 block steps and are means of 14 subjects; error bars are \pm 1 SEM. Times of MEP testing are denoted on the $x$-axis. MEPs are normalized to MEP amplitude measured at $B$. Conditioned MEP amplitudes were divided by test MEPs. Significant amplitude suppression was evoked at time points of intervention $\left(\mathrm{I}_{2}\right.$, $\mathrm{I}_{5-7}, \mathrm{I}_{11-13}, \mathrm{I}_{15-17}$ and $\mathrm{I}_{19-20}$ ) in APB muscle, contrary to post intervention effect those consistent differed from baseline. The asterisk marks a significant difference $(P<0.05$; Friedmann test, followed by the paired Wilcoxon test, post hoc analysis). All data are means \pm 1 SEM from 14 subjects. measures ANOVA was run with the within-subject effects TIME $\left(\mathrm{P}_{1}-\mathrm{P}_{6}\right)$ and the between-subject effect group (LTP, LTD).

Paired two-tailed t-test was applied for post-hoc analyses ( $p$ value was adjusted for the number of comparisons during post-hoc analyses). Effects were considered significant if $\mathrm{p}<0.05$. Results are given as means \pm SD.

Considering a potential differences in MEP amplitudes during interventional protocols Friedman twoway analysis of variance by ranks was performed, followed by Wilcoxon Signed Ranks tests for post-hoc testing.

\section{Results}

\section{Effects of interventional procedures on RMT}

RMTs were not affected by $\mathrm{PAS}_{\mathrm{LTP}}$ or $\mathrm{PAS}_{\mathrm{LTD}}$, registered immediately after intervention (ANOVA: $\mathrm{p}=0.42$ and $\mathrm{p}=0.41$, respectively).

\section{Effects of PASLTP and PASLTD on post-intervention} MEP amplitude

PAS $_{\text {LTP }}$ resulted in an expected increase in MEP amplitude in the APB from $0.984 \pm 0.257 \mathrm{mV}$ at baseline to an average of $1.401 \pm 0.389 \mathrm{mV}$ for time points $\mathrm{P}_{1}$ to $\mathrm{P}_{6}$ $(p<0.01)$, while PAS $_{\mathrm{LTD}}$ induced MEP amplitude inhibition starting from $0.859 \pm 0.251 \mathrm{mV}$ at baseline, to an average of $0.676 \pm 0.256 \mathrm{mV}$ for time points $\mathrm{P}_{1}$ to $\mathrm{P}_{6}$ $(p<0.01)$. Both induced effects lasted for at least $30 \mathrm{~min}$
(Figs 2 and 3). The overall MEP amplitude modulation for PAS $_{\mathrm{LTP}}$ and $\mathrm{PAS}_{\mathrm{LTD}}$, were $41.45 \pm 17.76 \%$ and $22.39 \pm 7.39 \%$, respectively.

\section{Interventions}

The Friedmann test showed a statistically significant global decrease for the MEP amplitudes during PAS $_{\text {LTP }}$ protocol $(\mathrm{p}<0.05)$ (Fig. 2), while suggestive trend of MEP amplitude increase during

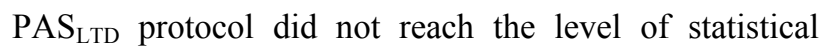
significance $(p=0.169)$ (Fig. 3). Further analysis by the paired Wilcoxon test showed a significant decrease at specific time points as assigned with asterisks (Fig. 2).

\section{Discussion}

The knowledge of cortical plasticity in humans is limited and mainly related to indirect data originated from non-invasive experiments based on different protocols involving TMS.

Among several models of inducing LTP- or LTD-like plasticity in the intact human brain, PAS protocol, based on the principle of Hebbian-like associative plasticity, has attracted most attention. The reason is that PAS protocol resembles invasive stimulation protocols applied at hippocampal tissue sections or those stimulation patterns, similar to bidirectional spike-timing dependent plasticity, that have been performed in animal models (Caporale and Day 2008). 


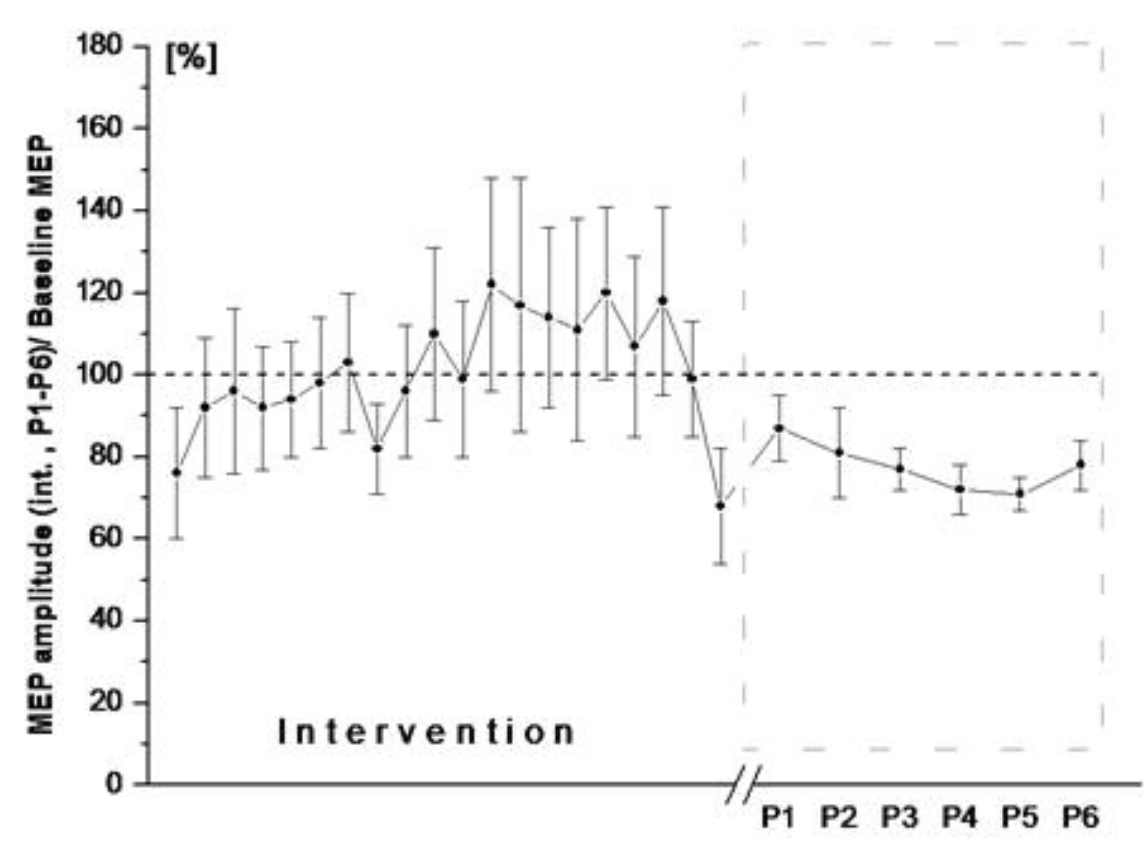

Fig. 3. Effects of $P A S_{L T D}$ on MEPS amplitude during intervention and post intervention in the resting abductor pollicis brevis (APB) muscle. Conventions and arrangement are the same as in Fig. 2.
However, previous neurophysiological studies in humans have also confirmed that LTP- or LTD-like plasticity in humans can be counterbalanced depending on the previous history of synaptic activity. It was implied that such a compensatory effect is intended to provide adjustment of overall synaptic weight and firing rate within the neural network, and within the physiological range. Those findings were confirmed if

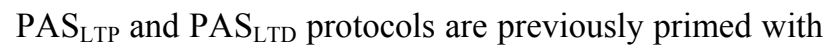
motor training, since in the former motor practice caused the cancellation of the effect of stimulation, while in the latter it was the opposite (Ziemann et al. 2004, Rosenkranz et al. 2007). Contrary to the above, if the sequence of motor activity and PAS was exchanged for each other replaced, homeostatic intervention was observed only in the case of LTD-like, but not in LTPlike plasticity (Jung and Ziemann 2009). However, these studies have analyzed the modulation of MEP amplitude in the post-intervention period, because the potential benefits in rehabilitation processes related precisely to this period. Conversely, if the research focus was directed towards the understanding of physiological processes that underlie the modulation of MEP amplitude, intervention period should be thoroughly analyzed, too.

Therefore, we focused our attention on MEP amplitude changes during PAS protocol, in order to resolve the paradox of the inverse MEP amplitude modulation during vs. after intervention. Our results have shown that PAS $_{\text {LTP }}$ protocol leads to MEP amplitude inhibition during the intervention protocol, opposite to subsequent typical MEP amplitude facilitation in postintervention period. Considering the $\mathrm{PAS}_{\mathrm{LTD}}$ protocol effects, despite the trend of MEP amplitude increase during intervention protocol, the statistically significant difference was not achieved, which may reflect the relatively small number of stimulation sessions. One possible explanation could be found in the mechanism of homeostatic plasticity, which is aimed to limit synaptic plasticity within the desired physiological range (Abraham and Bear 1996, Turrigiano and Nelson 2004). In this way, the history of previous activities of the motor system enables bidirectional modulation, or in other words, if the pre-induced MEP amplitude caused inhibition, the output from the system will subsequently meet with the facilitation, as it was destined to act within the framework of optimal values. These results indicate the complex nature of the interaction of activitydependent plasticity in relation to the temporal sequence of subsequent forms of activation, requiring further detailed experiments to explore cortical plasticity modulation under different conditions.

\section{Conflict of Interest}

There is no conflict of interest.

\section{Acknowledgements}

We acknowledge the support of the Ministry of Science and Technology of the Republic of Serbia project 175012 . 


\section{References}

ABOTT LF, NELSON SB: Synaptic plasticity: taming the beast. Nat Neurosci 3 (Suppl): 1178-1183, 2000.

ABRAHAM WC, BEAR MF: Metaplasticity: the plasticity of synaptic plasticity. Trends Neurosci 19: 126-130, 1996.

BIENENSTOCK EL, COOPER LN, MUNRO PW: Theory for the development of neuron selectivity: orientation specificity and binocular interaction in visual cortex. J Neurosci 2: 32-48, 1982.

BLISS TV, COLLINGRIDGE GL: A synaptic model of memory: long-term potentiation in the hippocampus. Nature 361: 31-39, 1993.

BOROOJERDI B, BATTAGLIA F, MUELLBACHER W, COHEN LG: Mechanisms influencing stimulus-response properties of the human corticospinal system. Clin Neurophysiol 112: 931-933, 2001.

CAPORALE N, DAN Y: Spike timing-dependent plasticity: a Hebbian learning rule. Annu Rev Neurosci 31: 25-46, 2008.

CHEN R, CROS D, CURRA A, DI LAZZARO V, LEFAUCHEUR JP, MAGISTRIS MR, MILLS K, RÖSLER KM, TRIGGS WJ, UGAWA Y, ZIEMANN U: The clinical diagnostic utility of transcranial magnetic stimulation: report of an IFCN committee. Clin Neurophysiol 119: 504-532, 2008.

CITRI A, MALENKA RC: Synaptic plasticity: multiple forms, functions, and mechanisms. Neuropsychopharmacology 33: 18-41, 2008.

CRUCCU G, AMINOFF MJ, CURIO G, GUERIT JM, KAKIGI R, MAUGUIERE F, ROSSINI PM, TREEDE RD, GARCIA-LARREA L: Recommendations for the clinical use of somatosensory-evoked potentials. Clin Neurophysiol 119: 1705-1719, 2008.

HEBB D: The Organization of Behavior: A Neuropsychological Theory. John Wiley and Sons, New York, 1949.

HESS G, DONOGHUE JP: Facilitation of long-term potentiation in layer II/III horizontal connections of rat motor cortex following layer I stimulation: route of effect and cholinergic contributions. Exp Brain Res 127: 279-290, 1999.

JUNG P, ZIEMANN U: Homeostatic and nonhomeostatic modulation of learning in human motor cortex. $J$ Neurosci 29: 5597-5604, 2009.

ROSENKRANZ K, KACAR A, ROTHWELL JC: Differential modulation of motor cortical plasticity and excitability in early and late phases of human motor learning. J Neurosci 27: 12058-12066, 2007.

ROTHWELL JC: Techniques and mechanisms of action of transcranial stimulation of the human motor cortex. J Neurosci Methods 74: 113-122, 1997.

SAKAI K, UGAWA Y, TERAO Y, HANAJIMA R, FURABAYASHI T, KANAZAWA I: Preferential activation of different I waves by transcranial magnetic stimulation with a figure-of-eight-shaped coil. Exp Brain Res 113: 24-32, 1997.

STEFAN K, KUNESCH E, BENECKE R, COHEN LG, CLASSEN J: Mechanisms of enhancement of human motor cortex excitability induced by interventional paired associative stimulation. $J$ Physiol 543: 699-708, 2002.

STEFAN K, KUNESCH E, COHEN LG, BENECKE R, CLASSEN J: Induction of plasticity in the human motor cortex by paired associative stimulation. Brain 123: 572-584, 2000.

TOKIMURA H, Di LAZZARO V, TOKIMURA Y, OLIVIERO A., PROFICE P, INSOLA A, MAZZONE P, TONALI P, ROTHWELL JC: Short latency inhibition of human hand motor cortex by somatosensory input from the hand. J Physiol 523: 503-513, 2000.

WOLTERS A, SANDBRINK F, SCHLOTTMANN A, KUNESCH E, STEFAN K, COHEN LG, BENECKE R, CLASSEN J: A temporally asymmetric Hebbian rule governing plasticity in the human motor cortex. J Neurophysiol 89: 2339-2345, 2003.

ZIEMANN U, ILIĆ TV, PAULI C, MEINTZSCHEL F, RUGE D: Learning modifies subsequent induction of longterm potentiation-like and long-term depression-like plasticity in human motor cortex. $J$ Neurosci 24: 16661672, 2004. 